Revue d'histoire de l'Amérique française

REVUE D.HISTOIRE DE L'AMÉRIQUE FRANÇAISE

\title{
Les Écrits attribués à Jacques Cartier
}

\section{Robert Le Blant}

Volume 15, numéro 1, juin 1961

URI : https://id.erudit.org/iderudit/302097ar

DOI : https://doi.org/10.7202/302097ar

Aller au sommaire du numéro

Éditeur(s)

Institut d'histoire de l'Amérique française

ISSN

0035-2357 (imprimé)

1492-1383 (numérique)

Découvrir la revue

Citer cet article

Le Blant, R. (1961). Les Écrits attribués à Jacques Cartier. Revue d'histoire de l'Amérique française, 15(1), 90-103. https://doi.org/10.7202/302097ar d'utilisation que vous pouvez consulter en ligne.

https://apropos.erudit.org/fr/usagers/politique-dutilisation/ 


\section{LES ÉCRITS ATTRIBUÉS À JACQUES CARTIER}

Il nous a été impossible de découvrir sur quel fondement l'un des plus récents historiens de Jacques Cartier a pu s'appuyer pour avancer que le malouin avait annoté la coutume de Bretagne. ${ }^{1}$ Les us et coutumes de la mer auraient en toute hypothèse été plus susceptibles d'intéresser le fameux pilote et nous ne pensons pas devoir discuter à nouveau des travaux rédigés avec une indifférence insuffisamment motivée pour les recherches dont le résultat a été publié en France. Les écrits pouvant être attribués à Jacques Cartier se trouvent donc réduits aux récits de ses voyages et aux signatures qu'il apposa sur des actes passés en sa ville natale.

Les dernières recherches sur Verrazano confirmant l'organisation italo-normande de ses expéditions ${ }^{2}$ et l'accoutumance des pêcheurs malouins sur les côtes du Labrador, notamment dans la région de Blanc-Sablon ${ }^{3}$ ne permettent guère de penser que le malouin aît accompagné Verrazano. Le premier récit de voyage concernant Jacques Cartier est donc celui de 1534.

\section{LE RÉCIT DU VOYAGE DE 1534}

Il a été publié dès 1556 en italien par Ramusio, ${ }^{4}$ puis, en anglais, par Halkluyt, ${ }^{5}$ en français par Raphaël du Petit-Val, ${ }^{6}$

1 Gustave Lanctot, Jacques Cartier devant l'Histoire (Montréal, 1947) $16^{\circ} \mathrm{Ln}^{27} 84035$, Bib. Nat., idem; Réalisations françaises de Cartier à Montcalm (Montréal, 1951) inconnu à la Bib. Nat.

2 Mollat, Le Commerce maritime normand à la fin du moyen-âge (Paris, Plon, 1952), 253-254, $8^{\circ} \mathrm{R} 55983$ à la Bib. Nat.; Anthiaume, Cartes marines, constructions navales, Voyages de découvertes chez les Normands, 1510-1650 (Paris, 1916), $8^{\circ}$ G 9840, id.

3 Jouon des Longrais, Jacques Cartier, documents nouveaux (Paris, 1888), $8^{\circ} \operatorname{Ln}^{27}$, 37453, Id.; R. Le Blant, Philippe de Pastour de Costebelle, gouverneur de Terre-Neuve (Paris, 1934), 119, $4^{\circ} \mathrm{Ln}^{27}$ 80352, Id.

4 Primo volume delle navigation, III. G 1453, idem.

5 Principal navigations etc., (London, 1598), Fol. G 349, idem.

6 Discours du voyage fait par le capitaine Jacques Cartier etc., Réserve $\mathrm{Lk}^{12} 717$, idem. 
édition utilisée par Lescarbot, ${ }^{7}$ réimprimée par Michelant, ${ }^{8}$ enfin par Guégan. ${ }^{9}$ Il existe d'autre part un manuscrit habituellement considéré comme un original, publié à plusieurs reprises, notamment par Michelant et Ramé, ${ }^{10}$ la Société historique de Québec ${ }^{11}$ en 1843, Biggar en 1924, ${ }^{12} \mathrm{~J}$. Pouliot en $1934 .{ }^{13}$ On en a même tiré une reproduction photo-mécanique ${ }^{14}$ et un fac-similé. ${ }^{15}$ Tout ne paraît pas avoir été dit sur ce manuscrit qui est conservé à la Bibliothèque Nationale au fonds Moreau sous le $\mathrm{N}^{\circ} 841$, fos. 52 à 58. Le texte est précédé d'un folio portant une suscription moderne: «Voiage de Jacques Cartier» et la date inexacte de 1544. L'écriture du document qui n'est pas signé est du XVIe siècle. Dionne, ${ }^{16}$ suivant Michelant et Ramé, a considéré que Jacques Cartier avait personnellement rédigé ce manuscrit et 3 arguments viennent à l'appui de cette opinion:

$1^{\circ}$ On trouve de temps à autre dans le texte un exposé à la première personne, exemple: «Je nomme cette isle Sainte-Catherine $\gg_{.}{ }^{17}$

$2^{\circ}$ Un passage du troisième voyage indique que Cartier aurait rédigé lui-même ses relations : « le roi ayant ouï ce qu'avait rapporté le capitaine Cartier, tant par ses écrits ... $\gg^{18}$

$3^{\circ}$ La même indication est donnée par une lettre de Jacques Nouel: «s'il y a moyen de retrouver ces relations que le capitaine Jacques Cartier a écrites $\gg .^{19}$

${ }^{7}$ Histoire de la Nouvelle-France, livre III, chap. 2 à 5, éd. Grant, t. 2, $8^{\circ} \mathrm{Pa} 78(7)$.

8 (Paris, Tross, 1865).

9 Trois Voyages au Canada (Paris, s.d.).

10 Relation originale, 1867 , in-16, $8^{\circ} \mathrm{Lk}^{12} 717$ ter, idem.

11 Voyages de découverte en Canada entre les années 1534 et 1542, $\mathrm{Lk}^{12} 718$, idem.

12 The voyages of Jacques Cartier (Ottawa), $8^{\circ} \mathrm{Pa} 71$ (II).

13 Glanures gaspésiennes - La grande aventure de Jacques Cartier (Québec), $8^{\circ} \mathrm{Pa} 352$.

14 (Paris, G. P. Maisonneuve, 1937), Bibliothèque de la Société historique du Canada, série bibliophile II, tiré à 150 exemplaires, inconnu à la Bibliothèque Nationale.

15 J. P. Baxter, A memoir of Jacques Cartier, sieur de Limoilon etc. (New-York, Dodd, Midd and Co, 1906), 464 p. in $8^{\circ}$, inconnu à la Bib. Nat. 16 Jacques Cartier (Québec, 1889), in-16, $8^{\circ} \mathrm{Ln}^{27} 39673$ p. 222; Michelant et Ramé, Relat. or., op. cit., introduction, p. IV.

17 Idem.

18 Dionne, op. cit., 243.

19 Idem. 
Si on examine le manuscrit, on remarque qu'au folio 64 vo., l'écriture devient beaucoup plus fine et plus compliquée. A défaut de pouvoir utiliser le fac-similé de Baxter ou la reproduction photo-mécanique, on peut le vérifier en comparant les photographies données par Biggar ${ }^{20}$ et par Pouliot..1 Sans toutefois constituer une preuve en faveur d'un original, cette modification de l'écriture donnerait à penser qu'il ne s'agit pas d'une copie, mais l'existence de cette catégorie de pièce ne pouvant être absolument écartée, le manuscrit peut donc avoir été rédigé par 2 personnes, copié par 2 personnes ou dicté à deux personnes. Il est en tout cas certain qu'une partie du document n'est pas de la main du découvreur. La paternité de la rédaction ne lui a pas été, du reste, attribuée par les augures susceptibles d'avoir étudié au moins les publications imprimées avant d'émettre une opinion. La «relation originale»a été habituellement attribuée à un certain Jehan Poulet à l'aide de raisonnements demeurés assez vagues, soit parce qu'il s'agit de l'œuvre d'un intellectuel, ${ }^{22}$ soit à l'aide d'un rapprochement avec le texte du $2 \mathrm{e}$ voyage. L'hésitation prudemment manifestée sur ce point par Biggar ${ }^{23}$ semble avoir pour origine une idée de Jouon des Longrais d'après laquelle ce Jehan Poulet aurait formé une compagnie pour l'organisation du premier voyage. ${ }^{24}$ Il est tout à fait vraisemblable qu'une méthode analogue à celle dont bénéficia Verrazano fut employée, mais on n'en trouve la trace ni pour Cartier, ni pour Roberval et nous avons vainement recherché le fondement sur lequel aurait pu être appuyée l'existence d'une telle intervention de Jehan Poulet. Plus importante est une remarque faite par d'Avezac, d'après laquelle le rédacteur du récit du $2 \mathrm{e}$ voyage aurait certainement fait partie du premier. ${ }^{25}$ Cette idée s'appuie

20 The Voyages ..., op. cit., 49.

21 Glanures, op. cit., 42.

22 De Cathelineau, « Jacques Cartier, Roberval et quelques uns de leurs compagnons », Revue des Questions historiques, (octobre, novembre 1934), $15,8^{\circ} \mathrm{G} 162$, à la Bib. Nat.

23 «all probability the redactor», The voyages, op. cit., renvoyant à The early Trading companies of New-France, 213 (Toronto, 1901), gr. in $8^{\text {? }}$, $4^{\circ} \mathrm{Z} 1195$, Hist. III, idem.

24 Jacques Cartier, op. cit., 127.

25 Brief récit, etc., (Paris, Tross, 1861), XV, Lk12 716, id. 
sur 2 passages du texte du $2 \mathrm{e}$ voyage qui paraissent décisifs: "Lequel cap cognoissons du précédent voyage », ${ }^{26}$ et «par les deux sauvages que avions prins le premier voyage $\gg .{ }^{27}$

Sans insister sur une intervention possible de Jehan Poulet, nous pensons qu'en dehors de la possibilité d'une dictée, qu'il s'agisse d'un original ou d'une copie, deux éventualités sont possibles : ou bien les rédacteurs ont tenu la plume pour Jacques Cartier, ou bien ils ont écrit, en son nom. Dans la première hypothèse, aucune difficulté n'apparaît, Jacques Cartier ayant fait les deux voyages dont les rédacteurs peuvent avoir été deux personnes différentes.

A l'appui de la seconde, on trouve: $1^{\circ}$ que Cartier est désigné à la troisième personne; $2^{\circ}$ que le rédacteur est vraisemblablement un ecclésiastique comme le donnent à penser une allusion à Caïn ${ }^{28}$ et l'emploi de la première personne du singulier pour baptiser certains lieux: «Je nomme cette isle Ste Catherine. » Celui de la première personne du pluriel en une circonstance serait en faveur de la participation de 2 prêtres à l'expédition: "et le dimanche, nous fimes chanter la messe ». Cette précision implique en tout cas la présence d'au moins un prêtre, l'officiant devant être distingué des fidèles et la célébration du saint sacrifice ne pouvant être confondue avec de simples prières. ${ }^{29}$

\section{LE RÉCIT DU 2ÈME VOYAGE (1535-1536)}

Le prétendu «brief récit et succincte narration de la navigation faicte, etc » a été imprimé dès 1545 en vertu d'un permis du dernier février 1544. On ne connaît que deux exemplaires de cette publication conservés l'un au British Museum, l'autre à la Bibliothèque Mazarine, à Paris.

D'Avezac l'a reproduite en édition critique, ${ }^{30}$ donnant les variantes de la publication et de 3 manuscrits, une adjonction

${ }^{26}$ Idem, 7.

27 Idem, 8, vo., Biggar, The Voyages, 102.

28 Biggar, idem, 22.

29 Idem, 24.

30 Brief récit, op. cit. 
importante d'après ceux-ci et d'autres variantes présentant un intérêt plus secondaire d'après des publications de Terneau Compan, ${ }^{31}$ de la Société littéraire et historique de Québec. ${ }^{32}$ Il n'apparaît nullement comme démontré que l'édition de 1545 aît été faite d'après un des manuscrits connus. Ces documents, conservés à la Bibliothèque Nationale à Paris, dans le Fonds français sous les Nos 5653, 5589 et 5644 ont été répertoriés par d'Avezac sous les lettres A.B.C., puis cet auteur a considéré que le manuscrit A pouvait être l'original présenté au roi parce qu'il ne comprend, ni une épître, ni des intitulés de chapitres qui se trouvent dans les autres manuscrits. ${ }^{33}$ D'Avezac, enfin, a indiqué qu'il s'agit de 3 expéditions d'un arché-type actuellement perdu, parce que l'écriture des 3 manuscrits est sensiblement la même. ${ }^{34}$ Dans ces conditions, il pouvait sembler inutile de continuer à épiloguer sur l'existence actuelle d'un manuscrit original. Cependant, l'érudit canadien Biggar a considéré que le manuscrit B était l'original ${ }^{35}$ en s'appuyant sur l'affirmation de Marc Lescarbot en vertu de laquelle cet auteur avait pris le texte publié par lui «sur l'original présenté au roy écrit à la main, couvert en satin bleu ». ${ }^{36}$ Il apparaît, en effet que A n'était pas recouvert de satin bleu en 1609, puisque nous le trouvons revêtu d'une reliure aux armes de Charles IX. Persuadé que Lescarbot a publié le manuscrit $B$ ayant déjà servi pour la première édition de 1545, Biggar a donc réédité le second voyage d'après le manuscrit $\mathrm{B}$ avec les variantes de l'édition de 1545 , celles des manuscrits $\mathrm{A}$ et $\mathrm{C}$, une traduction en anglais, des notes en anglais et un fac-similé du fo. 18.

Si nous nous reportons aux manuscrits, nous trouvons que: $\mathrm{I}^{\circ}$ le manuscrit $\mathrm{A}$ est rédigé d'une belle écriture du XVIe siècle très nette avec des abréviations et de belles majuscules, le texte paraissant avoir été écrit d'un seul jet. Il est abrité sous une belle

31 Archives des Voyages, 1841 (Paris), 2 vol., G 18994-5, Idem.

32 Voyages de découvertes, op. cit.

33 Brief recit, op. cit., 50.

34 Idem, 49.

35 The early, 213.

36 Histoire de la Nelle France, op. cit., 386. 
reliure marron à fleurs de lys, aux armes de Charles IX, comme nous le savons. Comprenant un intitulé ainsi conçu «Seconde navigation faicte par le commandement et voulloir du très chrétien roy », le texte est écrit sur du papier à verges séparées par un intervalle de $18 \mathrm{~mm}$ avec un filigrane représentant un pot à une seule anse surmontée d'une croix en forme de trèfle, variante dont nous n'avons pas retrouvé la reproduction dans Briquet. ${ }^{37}$ Le papier comportant ce filigrane à pot comprend un feuillet blanc suivant la fin du manuscrit et suivi lui-même de 3 autres feuillets blancs d'un papier dont les verges sont plus espacées. Le dernier feuillet comporte un filigrane représentant une sorte de $\mathrm{Q}$ à double trait transpercé par un trait. Le manuscrit est précédé de feuillets blancs d'un papier encore différent aux verges séparées par un intervalle de $22 \mathrm{~mm}$ muni d'un filigrane représentant une sorte de $R$ à double trait. En ce qui concerne le manuscrit, la première ligne de l'intitulé «Seconde navigation etc » est d'une écriture plus grosse. On relève des corrections, notamment au fo. 57, «blé gros comme des poix» et «ozisy 》 dans le dictionnaire, à la fin.

$2^{\circ}$ Le manuscrit $B$ dont l'écriture est différente de celle de A est précédé de feuillets blancs dont le papier aux verges moins serrées que celles du corps du manuscrit comporte un filigrane représentant des lettres majuscules. L'en-tête «Seconde navigation» est précédé d'une introduction adressée au roi très chrétien écrite sur le même papier que celui des feuillets blancs. Il en est de même pour l'intitulé «Seconde navigation». Le papier aux verges plus serrées avec le filigrane représentant un pot analogue à celui de $\mathrm{B}$ apparaît seulement à partir de « Le dimanche, jour et feste », c'est-à-dire du récit proprement dit.

$3^{\circ}$ Le manuscrit $\mathrm{C}$ comporte également une dédicace au roi très chrétien sur papier à verges écartées et sans filigrane. Il ne comporte pas d'en-tête «Seconde navigation». Comme au manuscrit $B$, le papier à verges serrées et muni d'un filigrane à pot n'apparaît qu'avec «Le dimanche, jour et feste ». L'écriture

37 Les Filigranes (Genève, 1907), in. f. t. IV, à la disposition des lecteurs à la réserve de la salle de travail de la Bib. Nat. et Folio Q 240, idem. 
n'est pas la même que celle de $B$ et diffère totalement de celle de A.

Nous croyons avec d'Avezac, mais parce que ces manuscrits sont calligraphiés, qu'ils sont trois copies d'un arché-type disparu. Nous considérons comme démontré par l'examen des papiers que le manuscrit original commençait seulement avec le récit du voyage «Le dimanche, jour et feste etc ». Le texte fut complété ensuite par un intitulé "Seconde navigation » ajouté aux manuscrits $\mathrm{A}$ et $\mathrm{B}$, une introduction au roi très chrétien introduite dans $\mathrm{B}$ et $\mathrm{C}$. On doit penser que le manuscrit présenté à François Ier comportait le maximum de manifestations de déférence, ce qui est le cas du manuscrit B. C'est donc bien celui-ci qui semble avoir rempli cet office, mais il s'agirait d'une copie établie dans ce but et non pas d'un original, vérité que Biggar aurait pu déduire du texte de Lescarbot après l'avoir rectifié grâce à des vérifications personnelles. Au demeurant, B est le seul parmi les manuscrits connus qui aît pu être reproduit par Lescarbot, puisqu'il est le seul à comporter l'épitaphe au roi et l'intitulé « Seconde navigation » publiés par l'avocat. ${ }^{38}$ On pourrait penser que $\mathrm{C}$ a fait l'objet de la publication de 1645 , puisqu'il ne comporte pas l'intitulé «Seconde navigation». Malheureusement, il se rapproche plus de $\mathrm{A}$ que du texte imprimé, tandis que l'intervention de Jean Poulet est en faveur de la reproduction d'un manuscrit perdu. ${ }^{39}$

\section{Les Signatures atTRIbuÉes À JACQUes CARTier}

F. Jouon des Longrais a reproduit jusque sur la couverture du livre qu'il a consacré au navigateur ${ }^{40}$ une signature en gros caractères ornée de 2 paraphes dont un très compliqué. Son attention ayant été attirée par la complexité du travail, aussi par certaines différences relevées entre les seings, le savant chartiste en a conclu que Jacques Cartier usait d'une signature impersonnelle à lui enseignée par un maître d'écriture résidant alors à Saint-Malo et nommé Armel Desgrés.

38 Op. cit., 411 et suiv.

39 D'Avezac, op. cit., 7 du texte.

40 Jacques Cartier, documents nouveaux, op. cit., 121. 
L'apparition d'une théorie admettant l'existence de signatures impersonnelles quoique très appliquées et ornementées peut causer quelqu'étonnement à priori. Le but recherché lors de l'application d'une signature au bas d'un acte consiste habituellement dans la recherche de l'établissement d'une preuve qui est celle de l'identité d'une partie ou d'un témoin. Presque toutes les signatures attribuées à Jacques Cartier figurent au bas des actes de baptême de Saint-Malo; elles paraissent donc avoir été réalisées dans le but de conserver la trace de la présence du célèbre pilote dont l'identité ne paraîtrait pouvoir être établie que par des signatures permettant de reconnaître qui en fut l'auteur. La personnalité d'une signature peut faire tout naturellement défaut au moins en apparence lorsque son auteur a opéré hâtivement ou négligemment, mais il est possible alors de retrouver la trace d'un «ductus» reconnaissable même dans un simple paraphe. De plus, en dépit de sa hâte ou de sa négligence, le signataire doit en principe tenter de sauvegarder un certain caractère de continuité même dans les paraphes; à défaut, ces derniers perdraient toute espèce d'utilité.

Des modifications importantes peuvent être causées dans les signatures par l'avancement de l'âge ou la maladie, mais les observations faites à propos des paraphes trouvent ici leur application: certaines caractéristiques du «ductus»doivent persister et l'absence d'une continuité tout au moins temporaire ne saurait s'expliquer. L'intervention d'une précipitation, d'une négligence, d'un âge ou d'une maladie doit donc être écartée lorsqu'on se trouve en présence d'un lot important de signatures espacées dans le temps, appliquées, ornementées et présentant des différences paléographiques évidentes. Nous savons d'autre part que certains personnages ont modifié totalement leurs signatures en remplaçant leur écriture gothique par une cursive. Marcel Delafosse en a signalé deux exemples, ceux du corsaire Guillaume Allène et du grand Guiton, ${ }^{41}$ mais il convient d'observer que le nouveau mode de signature présente un caractère d'analogie dans la continuité.

${ }^{41}$ M. Delafosse, Revue d'Histoire de l'Amérique française, op. cit. (septembre 1958), 12: 208. 
Il faut ajouter que Jouon des Longrais a rejeté l'hypothèse et même la possibilité de l'existence de signatures faites pour autrui simplement parce qu'il les a considérées comme absolument invraisemblables au bas d'actes aussi authentiques que des actes de baptême. Il est possible de faire observer que si l'on admet l'existence de signatures impersonnelles ne permettant pas d'identifier leur auteur, peu importe, assurément que de tels seings émanent d'une personne ou d'une autre. Quoi qu'il en soit, aucune objection n'aurait vraisemblablement été soulevée à l'encontre de la théorie admettant l'existence de signatures impersonnelles de Jacques Cartier si le révérend père Pouliot n'avait publié les photographies d'abord d'une signature sensiblement différente par le «ductus» et les paraphes de celle qui a été reproduite par Jouon des Longrais, ${ }^{42}$ puis d'un troisième seing totalement différent, indiqué comme figurant au bas d'un acte de baptême du 17 juillet 1530.43 D'autres dissemblances résultent de l'examen des fac-similés de 4 signatures conservés au fonds Margry à la Bibliothèque Nationale. ${ }^{44}$ Il paraît possible de les scinder en 2 familles ou groupes. Le premier comprend avec des différences dans les paraphes 2 signatures des 30 août 1529 et 17 juillet 1530 comportant une abréviation du prénom «Jacques » constituée seulement par un « $J$ » renforcé d'un exposant à sa droite. Le second groupe comprend 2 signatures abrégeant le prénom sous la forme «Jac». On pouvait se demander dans ces conditions si, même pour signer au bas des actes de baptême conservés dans les registres paroissiaux de Saint-Malo, Jacques Cartier n'avait pas fait tenir la plume par une personne plus apte qu'un simple pilote à réaliser des paraphes compliqués et pour continuer la discussion de la théorie avancée par Jouon des Longrais, un coup d'œil sur l'ensemble des signatures semblait indispensable.

Jacques Cartier assista pour la première fois à un baptême le 21 août 1510, mais sa première prétendue signature n'est que du 3 mars 1518. Cartier qui n'est pas parrain est porté comme

42 Glanures gaspésiennes, op. cit., 154.

43 Idem, 187.

44 Nelles Acq. françaises 9384, fo. 244, à la Bib. Nat. 
présent. ${ }^{45}$ Des caractéristiques très particulières apparaissent immédiatement: le « $\mathrm{J} \gg$ pourvu d'un exposant indiquant le prénom passe au travers $d u$ 《 $C$ », première lettre du nom. Le « $t$ » de Cartier oblique en haut à droite un peu comme un « $\mathrm{C} »$. A la droite du nom se trouve une sorte de dessin à la plume, tandis qu'un trait ornementé souligne le nom. La signature suivante sous un acte du 16 mars 1519 est analogue, sauf en ce qui concerne le trait soulignant le nom qui a disparu. Une signature du 5 juin 1519 continue la famille, mais le dessin à droite est très léger, tandis que la tête $d u$ 《 $J$ » présente un grand trait de plume fantaisiste dirigé vers la gauche. Le paraphe manque totalement. Des observations analogues se poursuivent: le 7 avril avant Pâques 1520, on trouve un très joli dessin à droite souligné d'un paraphe et quelques enjolivures sous le nom, tandis que la prolongation de la tête $d u$ 《 $J$ » vers la gauche est raisonnable. La signature du 20 janvier 1522 est celle qu'on a coutume de faire figurer dans les expositions comme particulièrement photogénique. Les principes ne sont pas modifiés, mais un caractère spectaculaire provient de l'allongement de la tête $d u$ 《 $J$ »ers la gauche, d'un signe d'abréviation sur le mot « présent » suivant le nom, d'un dessin sur la droite séparé par un paraphe d'une sorte de gribouillis fait de traits répétés prolongeant encore l'ensemble vers la droite. Il apparaît que les quatre signatures qui viennent d'être examinées sont issues d'une même main conduite par des préoccupations artistiques à des réalisations partiellement différentes sans souci déterminé d'ajouter ou de retrancher. Le scribe exécute suivant sa fantaisie des ornementations sur un corps de signature respecté, se préoccupant même, peut-être de ne pas rééditer exactement le même travail. Nous sommes loin de signatures impersonnelles, car le «ductus » et la méthode de l'artiste sont reconnaissables. Un changement paléographique d'une certaine importance apparaît avec la signature du pénultième d'avril 1529. ${ }^{46}$ Tandis qu'auparavant, la construction des $\ll \mathbf{r} »$ était à peu près celle que nous rencontrons de nos jours,

45 Registres paroissiaux de Saint-Malo, GG5, fo. 46 , actuellement aux Archives départementales d'Ille et Vilaine. $46 \mathrm{GG}^{6}$, fo. 46 , idem. 
nous trouvons ici les « $\mathrm{r} »$ arrondis fréquents au XVIe siècle, bien que le dernier $《 \mathbf{r} \gg$ de Cartier marque une hésitation entre les deux méthodes. D'autre part, le «a » de Cartier incline vers la scripture gothique et l'exposant en haut à droite $d u$ 《 $J$ » en est nettement séparé. Ces remarques sont plus pertinentes que celles qui portent sur les ornements: le $《 \mathrm{~J} »$ traverse le $《 \mathrm{C} »$, mais comporte 2 larges coups de plume en haut vers la gauche; un paraphe à double ornementation contribue à former un bel ensemble complété par un gribouillis à droite.

Dans la signature du 17 juillet 1530 reproduite par le père Pouliot, le $《 J 》 d u$ prénom traverse encore le $« \mathrm{C} \gg$ du nom avec 2 larges coups de plume vers la gauche et un paraphe précédé d'une rallonge $d u$ « $J »$ comportant un double ornement. Le nom Cartier est d'une grosse écriture droite avec un gribouillis à droite et un paraphe. ${ }^{47}$ Deux signatures des 24 et 26 septembre 1530 s'apparentent avec celle qui vient d'être décrite, mais une différence très importante et très complexe apparaît le 29 mai 1532. Deux signatures données ce jour s'apparentent avec les précédentes, mais diffèrent totalement de celles qui figurent au registre $\mathrm{GG}^{5}$. Il s'agit de l'aboutissement d'une évolution commencée le pénultième d'avril 1529 , se poursuivant au moins jusqu'au Ier décembre 1536, date à laquelle la mention «présent fut J. Cartier » s'apparente encore. Sans que nous puissions en tirer une déduction précise, il apparaît donc que l'existence d'une seconde famille puisse être relevée, évolutive même en ce qui concerne l'écriture, portant sur la période du dernier avril 1529 au Ier décembre 1536. L'ensemble de modifications le plus important et le plus brutal apparaît le 27 avril 1547 au-dessous de l'acte de baptême de Jacques Odiepvre. L'abréviation du prénom n'est plus réalisée par un simple « $J$ » renforcé d'un exposant, mais par les trois lettres «Jac ». ${ }^{48}$ La signature ne comporte pas de paraphe, $\mathbf{l}$ ' $\langle\mathbf{r} \gg$ final est totalement différent de celui de la signature précédente et on trouve à droite un véritable dessin représentant des espèces de fleurs.

$47 \mathrm{GG}^{6}$ 1525-1533, fo. 72 .

$48 \mathrm{GG}^{9}$. 
L'abréviation «Jac» se trouve reproduite le 8 décembre 1547 , le 29 mars 1548 , le 19 mars et le 10 novembre 1555. Il convient d'ajouter à ce groupe de signatures celle qui a été reproduite par Jouon des Longrais. Elle comporte un paraphe sous le nom et la particularité singulière d'un second paraphe sous un dessin à droite comprenant des espèces de 3 . Nous ne l'avons pas retrouvée dans les registres paroissiaux et Jouon des Longrais n'a pas indiqué sa provenance. ${ }^{49}$

Quelques difficultés nouvelles apparaissent avec une signature du 21 décembre 1547 qui se rapproche de celle qu'a publiée Jouon des Longrais en présentant un ductus analogue dans une écriture, cependant moins penchée, car elle est totalement différente des signatures qui la précèdent sur les registres paroissiaux. Des dessins sur le bas de l'acte au-dessus et au-dessous de la signature, un dessin supplémentaire à sa droite, un gribouillis très serré permettent de se demander si le scribe n'a pas eu l'idée d'ornementer l'acte de baptême. Les différences se multiplient avec une signature du 29 mars $1549 .^{50}$ Elle ressemble à celle du 8 décembre 1547, mais ne paraît pas être de la même main. Le caractère même relatif de continuité dans une même famille devient en tout cas fort douteux. Une série de variations générales apparaît encore le 20 octobre 1552: l'abréviation du prénom est réduite à peu de chose près aux deux lettres « Ja », tandis que l' « $\mathbf{r} »$ final ressemble à un «e $»$. Le nom et l'abréviation du prénom sont paraphés d'un seul trait et on ne trouve à droite qu'un gribouillis très serré. La continuité est loin d'avoir été rétablie le 10 novembre 1555. Nous retrouvons l'abréviation 《Jac », mais, tandis que l' $\langle\mathrm{r} »$ final ressemble à un «e $»$. Le 《 $\mathrm{J} \gg$ de $\ll \mathrm{Jac} »$ est fortement ornementé sur la gauche. Un autre ornement part $d u$ « $C$ »de Cartier pour former une boucle en bas à gauche. ${ }^{51}$

L'examen général des registres paroissiaux révèle l'existence au-dessus des actes d'inscriptions de noms de famille d'une écriture très simple et très lisible. Il ne s'agit pas de signatures

49 Jacques Cartier, op. cit., 121.

50 GG 10.

51 GG 11, fo. 22, vo. 
car ces inscriptions précèdent certainement les actes qu'elles concernent et il faut les considérer comme une nomenclature du XIXe siècle, vraisemblablement réalisée par l'abbé Paris-Jalobert. Les signatures ne constituaient pas une formalité substantielle car de nombreux actes n'en comportent aucune. On peut même constater l'absence de celle du prêtre Trublet. ${ }^{52}$ D'autre part, les signatures attribuées à Jacques Cartier ne sont pas seules à comporter des variantes importantes. La signature du prêtre «Gueret baptisant » en bas de l'acte de baptême du 27 novembre 1560 attribué à François Gravé, sieur du Pont ${ }^{53}$ est totalement différente d'une autre signature attribuable au même Guéret au bas de l'acte immédiatement précédent.

Il existe d'autres signatures artistiques du genre de celles qui ont été attribuées à Jacques Cartier, notamment celle de Guillaume Maingart au bas d'un acte du 23 février 1517 qui est gothique avec dessin à droite et trait de plume enjolivé au dessous. Ce sont les signatures de témoins qui constituent de tels chefs-d'œuvre: celles d'un certain Me Durocher sont aussi soignées que les signatures «Cartier». A la fin de $\mathrm{GG}^{5}$, le nom de Jourdain Le Roy figure dans un véritable petit tableau, tandis que des signatures «de Berthemont» présentent des analogies de conception avec celles qui nous intéressent, mais ne paraissent pas être de la même main. En résumé, les signatures attribuées au grand pilote se répartissent en 2 grandes familles distinguées par des abréviations différentes du prénom «Jacques ». Ces variantes ont un caractère de continuité dans les familles, mais on trouve à l'intérieur de celles-ci des sous-groupes comportant eux-mêmes des variantes parfois sans continuité et enchevêtrées. Les auteurs de ces signatures ont beaucoup moins cherché à les rendre personnelles qu'à poursuivre des buts artistiques ou spectaculaires. On doit les attribuer à au moins deux maîtres d'écriture, le premier ayant opéré avant le 27 avril 1647 et le second ensuite.

Pour le milieu du XVIe siècle, l'intention des auteurs de signatures en ce qui concerne les corps de ces écritures n'est pas

52 GG 6, fo. 70 .

$53 \mathrm{GG}^{13}$, à la date. 
claire: ou bien ils ont pu chercher à réaliser des signatures différentes, ou bien ils se sont contentés de reproduire certaines caractéristiques précédentes. On peut aussi supposer que l'intervention d'un nombre de signataires assez considérables s'est produite. L'ensemble de l'imbroglio pourrait peut-être trouver quelqu'éclaircissement dans un examen plus approfondi des corps de signatures en négligeant dans une certaine mesure les ornements auxquels nous avons peut-être attaché trop d'importance.

ROBERT LE BLANT

\section{MEMBRES DONATEURS}

Mgr J.-M. Phaneuf (Valleyfield) ………….......................... \$55.

Me René Chaloult (Québec) ……………………………………..... 50.

M. Bruno Lafleur (Québec) ………………………………... 50.

Thérien Frères Limitée (Montréal) ……………………………..... 50.

Mlle Jeanne Grégoire (Montréal) …………………………………... 50.

Dr Joseph Boulanger (Edmonton) ……………......................... 55.

Bureau des études et Ecole ménagère, SS. de l'Assomption de la S. V. (Nicolet)

Me Raymond Dupuis (Montréal)

30 .

Société historique du N.-Ontario

40 .

Mgr Olivier Maurault, p.s.s. (Montréal) ……………………………. 25.

M. l'abbé J.-E. Bernier (Montréal) ………………………………... 25.

Association c.-f. de l'Alberta …….................................................. 25.

La Ligue d'Action Nationale ………………………......................... 25.

Le Collège Sainte-Marie (Montréal) …………………………………... 25.

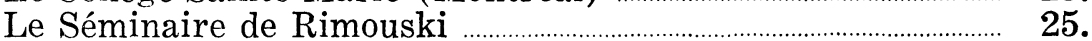

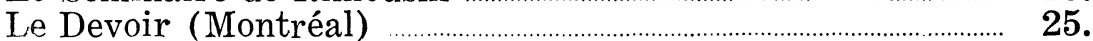

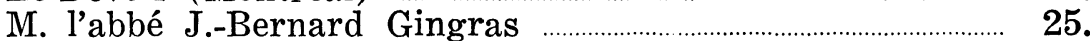

M. l'abbé Yvon Charron, p.s.s. (Montréal) ……………………….... 25.

M. le Chan. J.-H. Lecourt (St-Isidore de Laprairie) .................. 25.

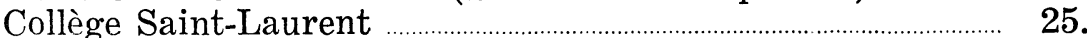

M. Robert Nadeau (Québec) …………………………………………....... 25.

M. Roland-J. Auger (Montréal) ……………………………………... 25.

Major (abbé) Emile Turmel (Ancienne Lorette) ……………..... 45.

M. l'abbé L. Sansoucy (Montréal) ……………………………………... 20.

Me Marie-Louis Beaulieu (Québec) ………………………………….... 20.

M. Jean-Robert Bonnier (Montréal) ………………………………….. 25.

M. Bernard Lanctôt (Montréal) ………………………………………….. 25.

M. Paul Lussier (Montréal) ........................................................................ 25. 